\title{
24-th Order High Temperature Expansion for the 3-d Ising Model* $^{*}$
}

\author{
Uwe Glässner, and Klaus Schilling** \\ Physics Department, University of Wuppertal Gaussstrasse 20, D-42097 Wuppertal, \\ Germany
}

Gyan Bhanot***

Institute for Advanced Study, Princeton, NJ 08540, USA

and

\section{Michael Creutz}

Physics Department, Brookhaven National Laboratory Upton, NY 11973, USA

\footnotetext{
* Talk presented by Uwe Glässner at the XII International Symposium on Lattice Field Theory, Bielefeld, Germany, Sept. 27-Oct. 1, 1994.

This work was supported in part by the grants Schi: 257/3-2 and 810/3-5 of Deutsche Forschungsgemeinschaft.

** also at HLRZ, c/o KFA, D-52425 Jülich, Germnay

*** also at IBM Watson Research Center, Yorktown Heights, ??

This manuscript has been authored under contract number DE-AC02-76CH00016 with the U.S. Department of Energy. Accordingly, the U.S. Government retains a non-exclusive, royalty-free license to publish or reproduce the published form of this contribution, or allow others to do so, for U.S. Government purposes.
}

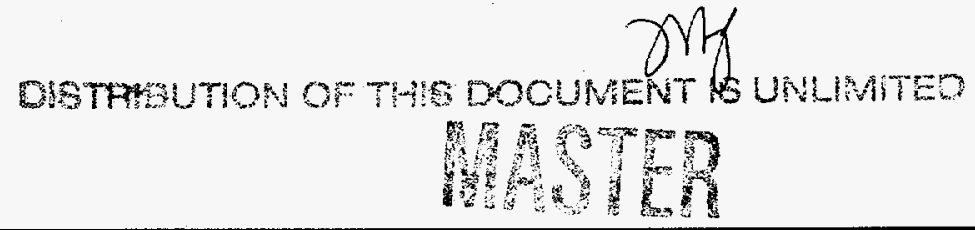


This report was prepared as an account of work sponsored by an agency of the United Stateis Government. Neither the United States Government nor any agency thereof, nor any of their employees, makes any warranty, express or implied, or assumes any legal liability or responsibility for the accuracy, completeness, or usefulness of any information, apparatus, product, or process disclosed, or represents that its use would not infringe privately owned rights. Reference herein to any specific commercial product, process, or service by trade name, trademark, manufacturer, or otherwise does not necessarily constitute or imply its endorsement, recommendation, or favoring by the United States Government or any agency thereof. The views and opinions of authors expressed herein do not necessarily state or reflect those of the United States Government or any agency thereof.

\title{
24-th Order High Temperature Expansion for the 3-d Ising Model
}

\author{
Uwe Glässner $^{\text {* *, Gyan Bhanot }}{ }^{\mathrm{bc}}$, Michael Creutz ${ }^{\mathrm{d}}$, and Klaus Schillingae* \\ apysics Department, University of Wuppertal Gaussstrasse 20, D-42097 Wuppertal, Germany \\ bInstitute for Advanced Study, Princeton, NJ 08540, USA \\ 'IBM Watson Research Center, Yorktown Heights, ?? \\ ${ }^{d}$ Brookhaven National Laboratory, Upton, NY 11973, USA
}

'HLRZ, c/o KFA, D-52425 Jülich, Germany

We present the series for the free energy and our estimate for the critical exponent $\alpha$, as computed by a recursive bookkeeping algorithm on the CM5.

\section{TRANSFER-MATRIX ALGORITHM}

We begin with a discussion of the algorithm to compute the High-Temperature(HT) expansion on finite 3-d Ising lattices. Starting from the action

$E\{s\}=-\sum_{\langle i, j\rangle} s_{i} s_{j}$,

the partition function is expanded in a HT series

$Z=\sum_{\{s\}} \exp (-\beta E)=\left(2 \cosh ^{3} \beta\right)^{V} \sum_{k} p(k) t^{k}$

with the expansion parameter $t=\tanh \beta . V$ is the volume of the system. The free energy per spin is defined as

$f=-\frac{1}{\beta V} \log Z=-\frac{2 \cosh ^{3} \beta}{\beta}-\frac{1}{\beta} \sum_{k} f_{k} t^{k}$.

For simplicity, consider a finite simple cubic lattice which, in the recursion algorithm, is built up by adding one site after the other, layer by layer. This procedure defines the recursion step, which requires knowledge only of those spin states that are contained in the exposed two-dimensional surface layer. To minimize finite size effects, it is best to use helical boundary conditions $[1,2]$. One can

*This work was supported in part by the grants Schi: 257/3-2 and 810/3-5 of Deutsche Forschungsgemeinschaft. visualize helical boundary conditions by imagining all spins in the layer laid out along a straight line. In this picture, the nearest neighbours to a given site in the sequence in the $i$ th direction can be chosen to be $h_{i}$ sites away, with $i=x, y, z$. It is convenient to assume $h_{x}<h_{y}<h_{z}$. It is easy to see that as spins are added, one needs only to keep track of the states of spins on the topmost $h_{z}$ sites. Let these spins be denoted $s_{1}, \ldots, s_{h_{x}}$. Then the partition function can be rewritten as

$Z \propto \sum_{k} \sum_{s_{1}, \ldots, s_{h,}} p\left(k ; s_{1}, \ldots, s_{h_{s}}\right) t^{k}$.

The recursion step, which consists of adding another spin $s_{0}$ to the system, can be can be carried out by perfornning the following updating procedure for the coefficients $p$ [4]

$$
\begin{aligned}
& 2 p^{\prime}\left(k ; s_{0}, s_{1}, \ldots, s_{h_{z}-1}\right) \\
= & p\left(k-0 ; s_{1}, \ldots, s_{h_{x}-1}, s_{0}\right) \\
+ & p\left(k-0 ; s_{1}, \ldots, s_{h_{z}-1}, s_{0}\right) \\
+ & p\left(k-1 ; s_{1}, \ldots, s_{h_{x}-1}, s_{0}\right)\left(s_{0} s_{h_{x}}+s_{0} s_{h_{y}}+1\right) \\
+ & p\left(k-1 ; s_{1}, \ldots, s_{h_{x}-1}, s_{0}\right)\left(s_{0} s_{h_{x}}+s_{0} s_{h_{y}}-1\right) \quad(5) \\
+ & p\left(k-2 ; s_{1}, \ldots, s_{h_{z}-1}, s_{0}\right)\left(s_{h_{x}} s_{h_{y}}+s_{0} s_{h_{x}}+s_{0} s_{h_{y}}\right) \\
+ & p\left(k-2 ; s_{1}, \ldots, s_{h_{x}-1, \overline{s_{0}}}\right)\left(s_{h_{x}} s_{h_{y}}-s_{0} s_{h_{x}}-s_{0} s_{h_{y}}\right) \\
+ & p\left(k-3 ; s_{1}, \ldots, s_{h_{x}-1}, s_{0}\right)\left(s_{h_{x}} s_{h_{y}}\right) \\
+ & p\left(k-3 ; s_{1}, \ldots, s_{h_{x}-1}, \overline{s_{0}}\right)\left(-s_{h_{x}} s_{h_{y}}\right) .
\end{aligned}
$$

It is crucial to remove finite-size errors by combining the results of different lattice structures as described in refs. $[1,2]$. We use the set of lattices 


\section{DISCLAIMER}

Portions of this document may be illegible in electronic image products. Images are produced from the best available original document. 
Table 1

Structures and weights $w$ of the lattices used

\begin{tabular}{|c|c|c|c|c|c|c|c|c|c|c|c|c|c|c|c|c|c|c|c|}
\hline$\overline{h_{x}}$ & 9 & $\overline{1}$ & $\overline{9}$ & 5 & 7 & 10 & 5 & 14 & 11 & 14 & 9 & 9 & $\overline{5}$ & 5 & 16 & 10 & 16 & $\overrightarrow{1}$ & 17 \\
\hline$h_{y}$ & 11 & 12 & 14 & 15 & 15 & 13 & 15 & 15 & 16 & 16 & 17 & 16 & 17 & 19 & 17 & 19 & 20 & 18 & 21 \\
\hline$h_{z}$ & 13 & 14 & 16 & 16 & 16 & 17 & 17 & 17 & 17 & 17 & 19 & 20 & 20 & 20 & 21 & 21 & 21 & 22 & 22 \\
\hline$w$ & -3 & 3 & -3 & -3 & 3 & -3 & 3 & -3 & 3 & 3 & -1 & -2 & -1 & $\overline{1}$ & -2 & 5 & 2 & -2 & 2 \\
\hline
\end{tabular}

Table 2

Free energy up to 24th order

\begin{tabular}{rr}
\hline order $\mathrm{k}$ & free energy $f_{n}$ \\
\hline 0 & 0 \\
2 & 0 \\
4 & 3 \\
6 & 22 \\
8 & $375 / 2$ \\
10 & 1980 \\
12 & 24044 \\
14 & 319170 \\
16 & $18059031 / 4$ \\
18 & $201010408 / 3$ \\
20 & $5162283633 / 5$ \\
22 & 16397040750 \\
24 & 266958797382
\end{tabular}

listed in table 1 and obtain the free energy coefficients up to 24th order as given in table 2 . In order to eliminate the contribution from (unphysical) loops with an odd number of links in any direction, we use the cancellation technique of ref. [2]. Possible contributions of higher-order finitesize-loops are at least of order 25 for this set of lattices. Since we use open boundary conditions, the coefficients $p$ are invariant under the global transformation $s_{i} \rightarrow-s_{i}$. This $\mathrm{Z}(2)$ symmetry enables us to reduce memory requirements by a factor of two. Unlike refs. [1-3] we use multipleword arithmetic to account for the size of the coefficients. This implementation needs about $100 \%$ more memory but leads to a doubling in performance. Since the number of words can be adjusted separately for every order, the computational effort can be reduced accordingly. On a 32 node Connection Machine CM-5 the total time for all computations was about 50 hours.

Compared to the finite-lattice approach of Enting and Guttmann [3], our method appears to require more CPU-time since we need to cancel unphysical loops. It should be noted, however, that helical lattices are very naturally implemented in data parallel software environments and thus lead to better performance. In the usual finite lattice method [3] on the other hand, the HT expansion can only be extended in fairly coarse steps, using lattices with $(4 \times 5)$ cross-section for 22 nd order and $(5 \times 5)$ cross-section for 26 th order. Thus a 24th order computation would not have been feasible with out 1 Gbyte machine.

\section{CRITICAL EXPONENT}

The specific heat is defined as

$\left.c\right|_{h=0}=\beta^{2} \frac{\partial^{2}}{\partial \beta^{2}} \log Z=\sum_{k} c_{2 k} t^{2 k}$

and is expected to behave near $T_{C}$ as

$\left.c\right|_{h=0}=A\left|T-T_{C}\right|^{-\alpha}\left[1+B\left|T-T_{C}\right|^{\theta}+\ldots\right]$, (7)

with $A$ and $B$ being analytic near $T_{C}[7,8]$.

We find for the critical exponent using inhomogeneous differential Padé-approximants (IDPs) [9]

$\alpha_{1}=0.102 \pm 0.008$

$\alpha_{2}=0.109 \pm 0.016$

at the value $t_{C}=0.218092$ obtained from MonteCarlo simulations [10]. The first value is obtained using a linear fit to the unbiased approximants [4], whereas the second value is the mean value from directly biased approximants.

IDPs can also be used to predict the most significant digits of the next term in the specific heat series [3]. The estimate of the 24th order term as 


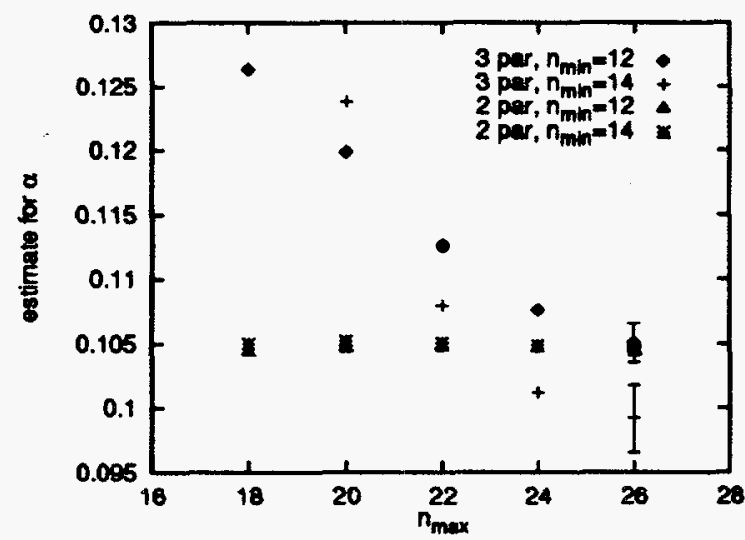

Figure 1. Estimates of $\alpha$ using 2- and 3parameter-fits. Each point represents the results of a fit to the set of values $\left\{s_{n_{\text {min }}}, \ldots, s_{n_{\text {mex }}}\right\}$. The error bars of the rightmost values represent the uncertainty of the extrapolated 26th term.

obtained in ref. [3] agrees perfectly with our exact result. In the same manner we can estimate the 26th order term in the expansion to be

$f_{26}=443762(4) \times 10^{7}$,

where the error quoted refers to two standard deviations.

The ratio $r_{n}=c_{n} / c_{n-2}$ of successive coeffcients of the specific-heat series is expected to behave as [7]

$r_{n}=\frac{1}{t_{C}^{2}}\left(1+\frac{\alpha-1}{n}+\frac{c}{n^{1+\theta}}+\frac{d}{n^{1+2 \theta}}+\ldots\right)$

Assuming that the correction-to-scaling exponent $\theta$ is close to $0.5[10,11]$, the following sequence $s_{n}$ is expected to converge towards $\alpha$ like

$s_{n}:=\left(t_{C}^{2} r_{n}-1\right) n+1=\alpha+\frac{c}{n^{1 / 2}}+\frac{d}{n}+\ldots$

We performed 3-parameter fits to the set of values $\left\{s_{12}, \ldots, s_{26}\right\}$ to obtain estimates for $\alpha$. The results of these fits are shown as diamonds in figure 1. To get an estimate of the uncertainties of our results, we investigate the stability of the fits by eliminating the point $s_{12}$ from the data. As a result we obtain sizeable changes for $\alpha$ shown as crosses in fig. 1.

Since we find that the correction-to-scaling coefficient $c$ vanishes within error we also performed 2-parameter fits to the same data set. The results of these fits are also shown in fig. 1

Finally we investigate the influence of the uncertainty in the correction-to-scaling exponent $\theta$ on our results. Repeating the analysis with $\theta=0.53[10]$ we find that the error in $\alpha$ is smaller than 0.0005 .

Taking into account the fact that omitting the $\frac{e}{n^{1 / 2}}$ gives rise to an additional systematic error, we endup with the estimate for the critical exponent

$\alpha=0.104(4)$.

\section{REFERENCES}

1. G. Bhanot, M. Creutz and J. Lacki, Phys. Rev. Letters 69 (1992) 1841; G. Bhanot, M. Creutz, I. Horvath, U. Glässner, J. Lacki, K. Schilling, J. Weckel, Phys. Rev. B48 (1993) 6183.

2. G. Bhanot, M. Creutz, I. Horvath, J. Lacki and J. Weckel, Phys. Rev. E49 (1994) 2445.

3. A.J. Guttmann and I.G. Enting, J.Phys. A26 (1993) 807.

4. G. Bhanot, M. Creutz, U. Glässner and K. Schilling, Phys. Rev. B49 (1994) 12909.

5. M. Creutz, Phys. Rev. B43 (1991) 10659.

6. K. Binder, Physica 62 (1972) 508; G. Bhanot, J. Stat. Phys. 60 (1990) 55; G. Bhanot and S. Sastry, J. of Stat. Phys. 90 (1990) 333.

7. A.J. Guttmann,Asymptotic Analysis of Power Series Expansions. In: C. Domb and J. Lebowitz (eds) Phase Transitions and Critical Phenomena, Vol. 13 (Academic Press, New York).

8. M.F. Sykes, D.S. Gaunt, P.D. Roberts and J.A. Wyles, J. Phys. A5 (1972) 624.

9. M. E. Fisher and H. Au-Yang, J. Phys. A (1979) 1677 ; D. L. Hunter and G. A. Baker, Phys. Rev. B 19 (1979) 3808.

10. C.F. Baillie, R. Gupta, K.A. Hawick and G.S. Pawley, Phys. Rev. B45 (1992) 10438.

11. A.J. Liu and M.E. Fisher, J. Stat. Phys. 58 (1990) 431. 